\title{
Continuous non-invasive cell growth monitoring in disposable bioreactors using an active compensated differential transformer
}

\author{
Maria Allers, Sven Schönewald, Kai Bakes, Stefan Zimmermann \\ Leibniz Universität Hannover, Institute of Electrical Engineering and Measurement Technology, \\ Department of Sensors and Measurement Technology, Appelstr. 9A, 30167 Hannover, Germany \\ E-Mail: allers@geml.uni-hannover.de
}

\begin{abstract}
Summary
In this work, we present a sensor system for continuous non-invasive cell growth monitoring, especially designed for single use bioreactors (SUBs). The sensor system is based on a differential transformer. In theory, using this differential setup, the influence of the primary magnetic flux is fully eliminated from the sensor signal and the sensor signal is zero for an ideally symmetrical, unloaded sensor. In practice, due to small geometric asymmetries and resonance effects, the sensor signal of the unloaded sensor can have a significant offset. Thus, complex measuring electronics is required to detect the small signal variations caused by permittivity changes in the cell culture medium. In order to use simple, low-cost electronics a new method for zero balancing of the sensor signal is presented. This method is based on an additional axially arranged compensation coil. Different mixtures of isopropanol and water were used as test liquids with defined permittivity and to evaluate the sensor system. Our results show that the additional compensation coil does not affect the sensitivity of the sensor system. Thus, a low-cost sensor system with low-cost electronics can be realized.
\end{abstract}

Keywords: cell growth monitoring, single use bioreactor, inductive permittivity measurement, differential transformer, zero balancing, flux compensation

\section{Introduction}

In order to obtain high-quality products from biotechnological processes, it is necessary to monitor all relevant process parameters. In modern biotechnology, bioprocesses are increasingly run in single-use bioreactors (SUBs). These disposable reactors offer a variety of advantages, such as high flexibility, low purchase and energy costs, and especially a lower risk of contamination, as SUBs are presterilized by gamma radiation [1].

During cell cultivation, biomass is one of the most important parameters, which has to be continuously monitored, as it describes the process performance, the process state and the condition of the cells. However, classical methods and sensors for measuring biomass are not really applicable to SUBs, since they usually have to be introduced into the presterilized reactor or sampling is required for an external off-line measurement [2-5].

In this work, an innovative approach for continuous non-invasive monitoring of cell growth, especially suitable for SUBs, is presented. Hereby, the sensor is simply attached to the SUB from the outside and biomass is non-invasively measured through the polymer foil of the SUB. This allows accurate biomass quantification without any risk of process contamination and multiple use of the sensor system. Furthermore, it is possible to implement the sensor independently of the manufacturer of the SUB. Since biomass is measured via an inductively induced electrical field to determine the complex permittivity of the cell culture as a measure for the biomass just living cells are taken into account, which is a major benefit compared to optical methods.

Capacitive sensors for dielectric spectroscopy show good results when the sensors are submerged in the culture medium [6]. However, they are not suitable for non-invasive monitoring of the cell density through the polymer foil of SUBs as the foil significantly lowers the electrical field strength in the culture medium, leading to reduced sensitivity. Since the foil does not disturb the magnetic flux density, a measuring principle based on magnetic induction is preferable for SUBs, and presented in this work. A primary coil is used to generate a primary magnetic field inducing an electrical field inside the culture medium. The 
measuring effect is the cell density dependent repercussion of the secondary magnetic flux. Since the sensor signal is dominated by the primary flux, the small secondary flux from the medium is hard to detect. Therefore, a differential setup is necessary that fully eliminates the primary flux from the sensor signal. Such a differential transformer [7] seems to be the most promising approach for non-invasive cell growth monitoring. Preliminary measurements show a good correlation between the biomass concentration in the cell culture medium and the sensor signal [7]. However, due to high offset voltages caused by geometric asymmetries and resonance effects, complex measuring electronics is required for the detection of small sensor signal variations caused by permittivity changes of the cell culture medium [8]. Here, we present a very promising approach to compensate such disturbing offset voltages without loss of sensitivity allowing the use of simple measuring electronics and eventually a low-cost sensor.

\section{Experimental}

As shown in Figure 1, our first differential transformer consists of three coils stacked on a ferrite core $\left(8 \mathrm{~mm}\right.$ in diameter, $\mu_{r}=300$, Frequency $0.1-3 \mathrm{MHz}$ ). The coils are wound with an insulated copper wire. The receiver coils $L_{s 1}$ and $L_{s 2}$ have a similar inductance of $L_{s}=10 \mathrm{mH}$ (2 $\mathrm{mm}$ high, $33 \mathrm{~mm}$ in diameter). In order to reach an increased sensitivity, a high turn ratio is realized by choosing a 100 -fold lower inductance for the excitation coil $\mathrm{L}_{p}=0.1 \mathrm{mH}$ (10 $\mathrm{mm}$ high, $15 \mathrm{~mm}$ in diameter) [8].

The two outer receiver coils $L_{s 1}$ and $L_{s 2}$ are connected in series but wounded in opposite directions, so that the primary flux generated by the centered excitation coil $L_{p}$ should not affect the measuring voltage $U_{s}$. The primary flux induces an electrical field inside the cell culture medium which leads to electrical currents: eddy currents, which depend on the conductivity of the cell culture medium and displacement currents, that depend on the complex permittivity of the cell culture medium. The secondary magnetic flux generated from these two currents finally induces a voltage, mainly in the receiver coil $L_{s 2}$ as it is placed much closer to the medium than the receiver coil $L_{s 1}$. The induced voltage in $L_{s 1}$ can be neglected. Therefore, the measuring voltage $U_{s}$ equals the induced voltage in Ls2. In [7], it is shown that the real part of the measuring voltage $U_{s}^{\prime}$ just depends on the polarization properties of the cell culture medium $\left(\varepsilon_{r}^{\prime}\right)$ and the imaginary part of the measuring voltage $U_{s}$ " depends on the dielectric losses $\left(\varepsilon_{r}\right.$ ") and the conductivity $(\mathrm{K})$.

The measuring voltage $U_{s}$ is zero for an ideally symmetrical, unloaded sensor, as the primary magnetic flux passes through identically both receiver coils. However, due to small geometric asymmetries and resonance effects, caused by parasitic coupling between the coils the measuring voltage $U_{s}$ is not fully zero balanced in real differential transformers [8].

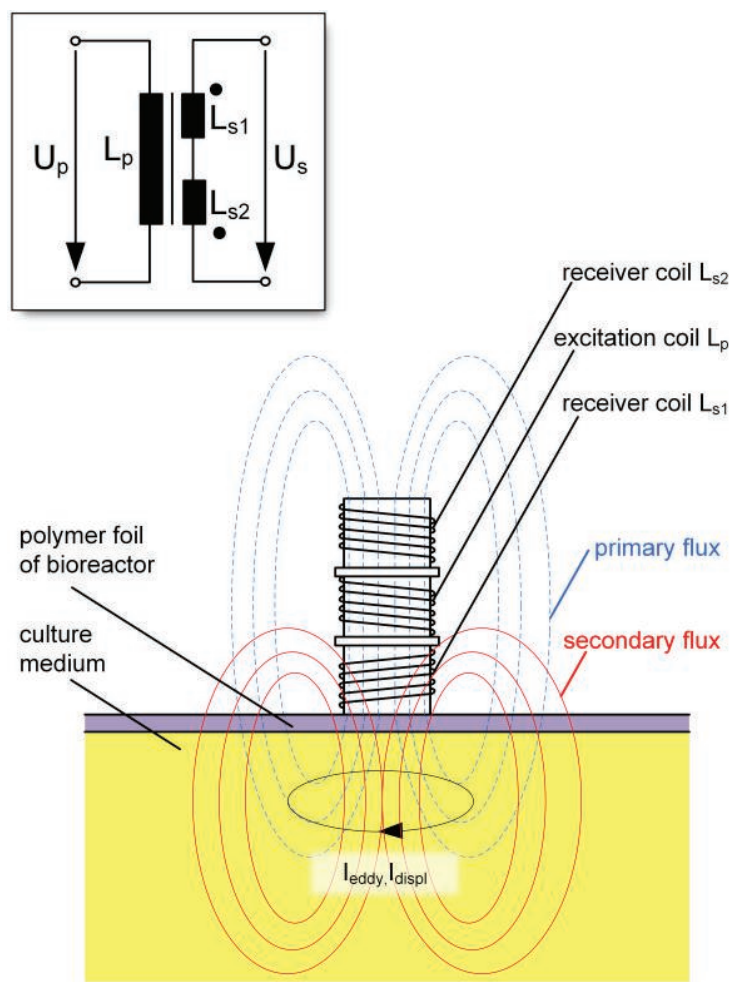

Fig. 1: Schematic of the differential transformer for continuous noninvasive cell growth monitoring in single use bioreactors.

As shown in Figure 2, in the unloaded state, the real part of the measuring voltage $U_{s}$ ' significantly exceeds zero depending on the frequency. Detecting even smallest permittivity variations of the cell culture medium requires complex measuring electronics with a wide measuring range and a high vertical resolution to accurately measure millivolts despite high offset voltages in the range of several volts.

In the current setup, an Agilent MSO9404A oscilloscope is used. For a low-cost sensor, this oscilloscope has to be replaced by simple electronics. Therefore, better zero balancing of the measuring voltage is necessary. 


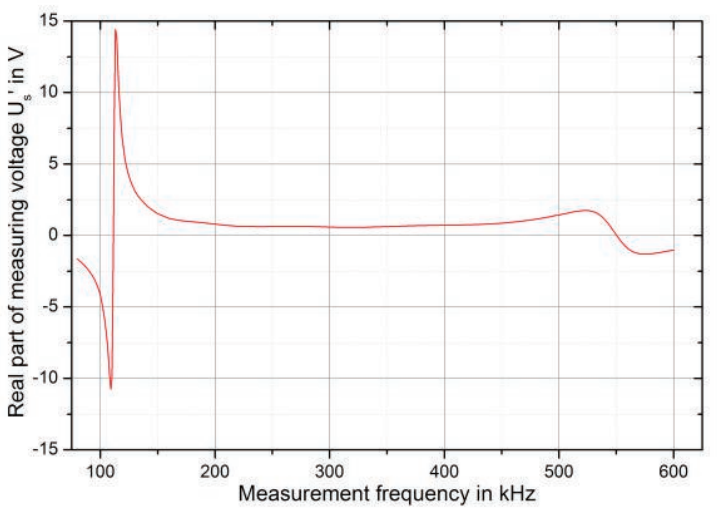

Fig. 2: Real part of the measuring voltage $U_{s}$ ' over frequency. Due to resonance effects the measuring voltage significantly exceeds zero at certain frequencies.

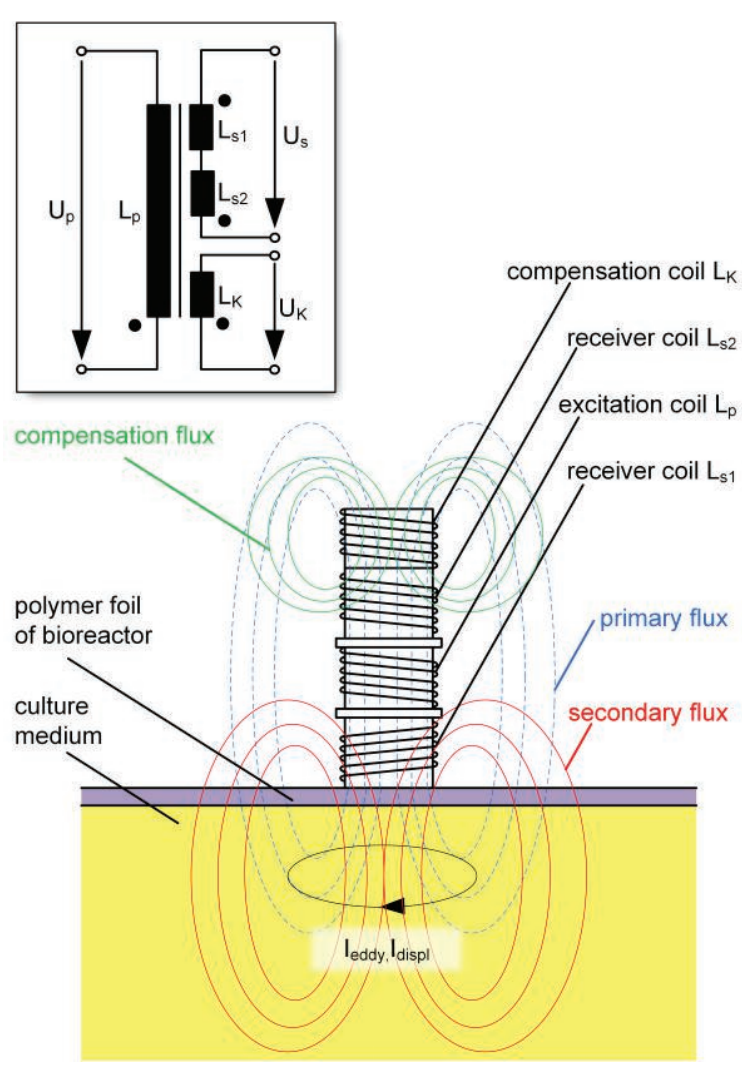

Fig. 3: Schematic of the new differential transformer with an additional compensation coil $L_{K}$ for better zero balancing.

According to [7], zero balancing of the measuring voltage can be achieved by vertical displacement of the outer receiver coil $L_{s 2}$. However, this also affects the parasitic coupling between the coils and shifts the resonance frequencies which can lead to decreased sensitivity when operating at a fixed measuring frequency [8]. Hence, we developed another zero balancing method of the measuring voltage. As shown in Figure 3, an axially arranged compensation coil $L_{k}$ is added. Easy zero balancing is now possible by applying the compensation voltage $U_{K}$.

For a certain measuring frequency, amplitude and phase of this compensation voltage can be adjusted to effectively reduce the measurement voltage offset to a minimum. Figure 4 shows the measuring voltage amplitude for different amplitudes and phases of the compensation voltage $U_{\kappa}$ at a measuring frequency of $400 \mathrm{kHz}$.

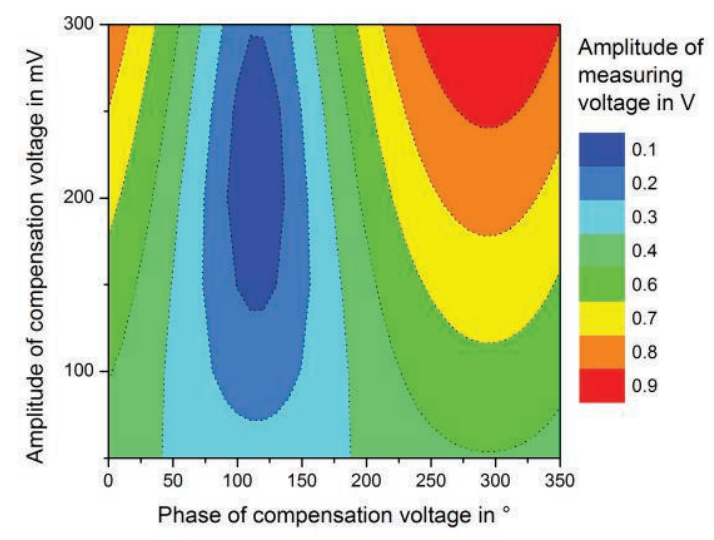

Fig. 4: Amplitude of the measuring voltage $U_{s}$ for different amplitudes and phases of the compensation voltage $U_{K}$ at a measuring frequency of $400 \mathrm{kHz}$.

Thus, by superimposing the generated compensation flux with the primary flux, small geometric asymmetries can be easily compensated leading to a significantly improved zero balance, see Figure 5 .

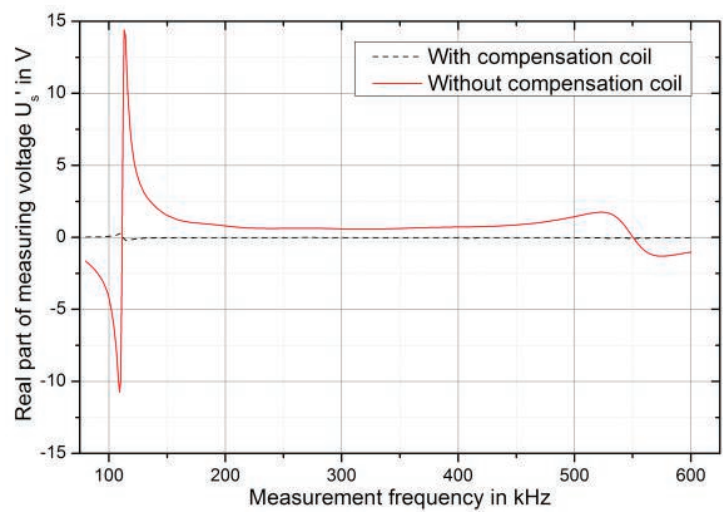

Fig. 5: Real part of the measuring voltage $U_{s}^{\prime}$ over frequency. Using the additional compensation coil the measuring voltage can be almost perfectly zero balanced. 


\section{Results}

The new sensor with optimized zero balancing is tested with different water-isopropanolmixtures. The permittivity values of such mixtures were calculated as described in [9]. In Figure 6 , the real part of the measuring voltage is plotted over the relative permittivity for the both sensor setups (with and without additional compensation coil). The absolute values of the real part of the measuring voltage are comparable. Thus, using an additional compensation coil significantly improves zero balancing without decreasing sensor sensitivity. Hence, it is now possible to realize a low-cost sensor since low-cost measuring electronics can be used with optimized zero balancing.

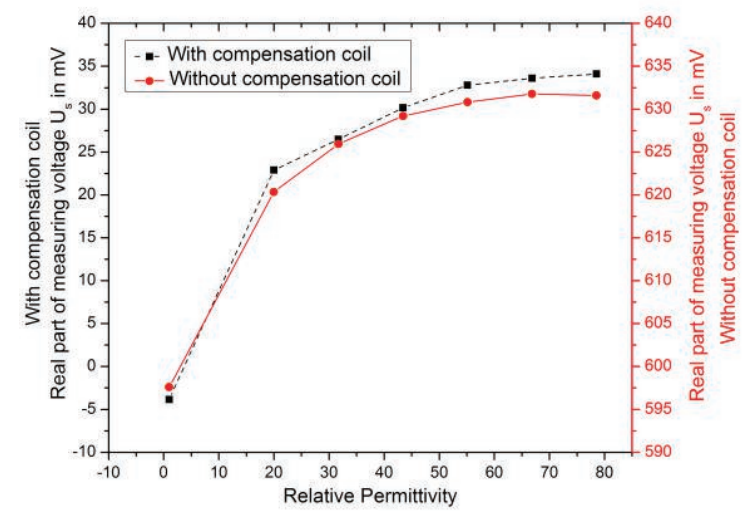

Fig. 6: Real part of the measuring voltage $U_{S}$ ' as a function of the relative permittivity. At a constant measuring frequency of $300 \mathrm{kHz}$ both setups show similar absolute voltage values.

\section{Conclusion}

In this work, we presented a new approach for continuous non-invasive cell growth monitoring during cell cultivation in single use bioreactors (SUBs) using a simple differential transformer. The setup consists of three coils stacked on a ferrite core. Significantly improved zero balancing of the measuring voltage is achieved by applying a compensation voltage to an additional axially arranged compensation coil. This allows sensitive detection of smallest variations of the measuring voltage with simple low-cost measuring electronics leading to a low-cost sensor for continuous non-invasive cell growth monitoring.

\section{References}

[1] R. Eibl, S. Kaiser, R. Lombriser, D. Eibl, "Disposable bioreactors: the current state-ofthe-art and recommended applications in biotechnology", Appl. Microbiol. Biotechnol. 86 (1), 41-49 (2010); doi: 10.1007/s00253-0092422-9

[2] S. M. Palmer, E. R. S. Kunji, "Online monitoring of biomass accumulation in recombinant yeast cultures", Methods in molecular biology (Clifton, N.J.), 866, 165-179 (2012); doi: 10.1007/978-161779-770-5_14

[3] B. Sonnleitner, "Automated Measurement and Monitoring of Bioprocesses: Key Elements of the M3C Strategy". In: Mandenius CF., Titchener-Hooker N. (eds) Measurement, Monitoring, Modelling and Control of Bioprocesses. Advances in Biochemical Engineering/Biotechnology, vol 132. Springer, Berlin (2012); doi: 10.1007/10_2012_173

[4] B. J. Downey, L. J. Graham, J. F. Breit, N. K. Glutting, "A novel approach for using dielectric spectroscopy to predict viable cell volume (VCV) in early process development", Biotechnology progress, 30 (2), 479-487 (2014); doi: 10.1002/btpr.1845

[5] J. P. Carvell, J. E. Dowd, "On-line Measurements and Control of Viable Cell Density in Cell Culture Manufacturing Processes using Radio-frequency Impedance", Cytotechnology, 50 (1-3) 35-48 (2006); doi: 10.1007/s10616-005-3974-x

[6] T. Reinecke, P. Biechele, V. Schulte, T. Scheper, S. Zimmermann: "Low-cost Sensor System for Non-Invasive Monitoring of Cell Growth in Disposable Bioreactors", Eurosensors XXIX, Procedia Engineering, 120, 548-551, Freiburg, Germany, September 2015; doi: 10.1016/j.proeng.2015.08.712

[7] T. Reinecke et al., "Continuous noninvasive monitoring of cell growth in disposable bioreactors", Sens. Actuators B: Chem., 251, 1009-1017 (2017); doi: 10.1016/j.snb.2017.05.111

[8] M. Allers, T. Reinecke, T. Nagraik, D. Solle, K. Bakes, M. Berger, T. Scheper, S. Zimmermann, "Differential inductive sensor for continuous non-invasive cell growth monitoring in disposable bioreactors", Eurosensors 2017, Proceedings, 1(4), 518, Paris, September 2017; doi: $10.3390 /$ proceedings 1040518

[9] A.P. Gregory, R.N. Clarke, "Tables of the Complex Permittivity of Dielectric Reference Liquids at Frequencies up to $5 \mathrm{GHz}$, NPL, 2001. 